EPiC Series in Engineering
Volume 3, 2018, Pages 1505-1512
HIC 2018. 13th International
Conference on Hydroinformatics

\title{
Increasing accuracy in shallow water flows: maintaining vorticity in presence of bathymetry
}

\author{
A. Navas-Montilla ${ }^{1 *}$ and J. Murillo ${ }^{1}$ \\ ${ }^{1}$ Universidad de Zaragoza, María de Luna 3, 50003, Zaragoza, Spain \\ anavas@unizar.es, Javier.Murillo@unizar.es
}

\begin{abstract}
The Kelvin-Helmholtz instability promotes the appearance of complex turbulent flow patterns which typically arise in presence of shear layers. Such condition evolves linear perturbations into complex turbulent structures that determine the mass exchange across the different jets/layers in the flow. In order to accurately predict the mass exchange across a shear layer in shallow flows over complex bathymetries, very high order numerical schemes are required. In this work, a WENO-ADER Augmented scheme, called ARL-ADER method, is used to provide accurate predictions of mass exchange processes under a variety of $2 \mathrm{D}$ scenarios involving turbulent shear layers. The proposed scheme is assessed in terms of numerical diffusion by means of the analysis of the numerical energy cascade. Numerical results show that the diffusivity of the scheme determines the turbulent structures produced along the shear layer. It is shown that traditional 1-st order schemes are very diffusive and would require the use of very fine meshes, which is computationally inefficient. On the other hand, the ARLADER scheme is able to capture the small-scale vortices and reproduce the theoretical energy cascade even in coarse meshes, while preserving the well-balanced property.
\end{abstract}

\section{Introduction}

In order to accurately assess the mass exchange across a shear layer in shallow flows over complex bathymetries, powerful numerical schemes are required. Such schemes must provide a high order of accuracy both in space and time in order to capture the complex turbulent flow pattern that appears in the mixing layer. The aim of this work is to study the numerical performance of an arbitrary order augmented ADER scheme for the resolution of the Kelvin-Helmholtz instability. This phenomenon occurs when a perturbation is introduced in a continuous fluid system composed of two (or more) jets at different velocities. Due to the inherent instability of the flow across the shear layer, the initial perturbation is amplified and evolves into a hydrodynamic two-dimensional turbulence (San

*Corresponding author: anavas@unizar.es 
\& Kara, 2015). The mass exchange across the layers is governed by such turbulent pattern and only very high order numerical models, that produce low dissipative solutions, can provide trustworthy predictions of such process in an affordable time.

Finite Volume (FV) numerical schemes are a very common choice for the resolution of complex flows of hyperbolic nature. Such schemes have recently experienced a great improvement thanks to the development of powerful methods that provide a high order of accuracy both in space and time.

Among those schemes, we can find the WENO-ADER methods. They are fully discrete and composed of two main steps: (1) an arbitrary order spatial reconstruction of the variables using the WENO method, which prevents from the generation of spurious oscillations avoiding the Gibbs phenomenon and (2) the updating of the variables in time by means of a one-step updating equation. The ADER methodology is based on the resolution of an arbitrary order extension of the traditional Riemann problem (RP), called Derivative Riemann problem (DRP).

WENO-ADER schemes have a wide range of applications in the field of continuum mechanics, when dealing with systems of hyperbolic conservation laws (Toro \& Titarev, 2002). In this work, it is of particular interest the application of such schemes to geophysical problems, especially for the resolution of the Shallow Water Equations (SWE) (Castro, Toro, \& Käser, 2012; Vignoli, Titarev, \& Toro, 2008)

The 2D SWE is given by

$$
\frac{\partial \mathbf{U}}{\partial t}+\frac{\partial \mathbf{F}(\mathbf{U})}{\partial x}+\frac{\partial \mathbf{G}(\mathbf{U})}{\partial y}=\mathbf{S}_{b}+\mathbf{S}_{f}
$$

where $\mathbf{U}=(h, h u, h v)^{T} \quad$ are the conserved variables, $\quad \mathbf{F}=\left(h u, h u^{2}+\frac{1}{2} g h^{2}, h u v\right)^{T}$ and $\mathbf{G}=\left(h v, h u v, h v^{2}+\frac{1}{2} g h^{2}\right)^{T}$ are the physical fluxes in the $\mathrm{x}$ and $\mathrm{y}$ directions and $\mathbf{S}_{b}=\left(0,-g h \frac{d z}{d x},-g h \frac{d z}{d y}\right)^{T}$ and $\mathbf{S}_{f}=\left(0,-c_{f}|\mathbf{v}| u,-c_{f}|\mathbf{v}| v\right)$ are the source terms accounting for variations in the bed level and friction. When designing the numerical scheme, the numerical treatment of source terms is a very challenging task. Source terms often dominate the dynamics of the solution and a suitable discretization procedure must be chosen in order to ensure convergence to a physically based solution.

In this work, a 2D extension of the ARL-ADER scheme presented in (Navas-Montilla \& Murillo, 2016) is used to compute mass exchange processes under a variety of scenarios involving turbulent shear layers. Such scheme uses an augmented Derivative Riemann Solver, called Linearized FluxSource (LFS) solver (Navas-Montilla \& Murillo, 2016), which allows to adequately include the presence of the bed variations. The method is herein assessed in terms of numerical diffusion using the analysis of the numerical energy cascade

\section{Materials and methods}

Following the FV approach, to compute the system in (1.1)-(1.3) inside the domain $\Omega \times[0, T]$, with $\Omega=[a, b] \times[c, d]$ the spatial domain and $T$ the final time, we discretize the computational 
domain into volume cells $\Omega_{i j}$ and integrate the variables and equations inside them. In this work, we will only consider Cartesian grids. The domain $\Omega$ is discretized in $N_{x} \times N_{y}$ volume cells.

If considering a regular Cartesian grid, all cells have a constant cell area $\Delta x^{2}$ and we obtain the following fully-discrete updating formula

$$
\mathbf{U}_{i j}^{n+1}=\mathbf{U}_{i j}^{n}-\frac{\Delta t}{\Delta x^{2}}\left(\sum_{r=1}^{4} \mathcal{F}_{r}^{-}-\overline{\mathbf{S}}_{i j}\right)
$$

where

$$
\overline{\mathbf{S}}_{i j} \approx \frac{1}{\Delta t} \int_{0}^{\Delta t} \int_{x_{i+1 / 2}}^{x_{1-1 / 2}} \int_{y_{i+1 / 2}}^{y_{t-1 / 2}} \mathbf{S} d y d x d \tau
$$

is the approximation of the space-time integral of the source term inside the cell and $\mathcal{F}_{r}^{-}$is the space-time integral of the numerical fluxes over the $r$-th cell edge. To construct a numerical scheme of order $K+1$-th, it is sufficient to approximate the integral of the flux, $\mathcal{F}_{r}^{-}$, using a $K+1$-th order Gaussian quadrature rule as

$$
\mathcal{F}_{r}^{-}=\frac{\Delta x}{2} \sum_{q=1}^{k} w_{q} \mathcal{F}_{r, q}^{-}
$$

where $w_{l}$ are the Gaussian weights inside the interval $[-1,1]$ at the $q=1, \ldots, k$ quadrature points along the cell edge and $\mathcal{F}_{r, q}^{-}$the numerical fluxes at each of these points, computed by means of the resolution of the DRP with at least $K$ non-trivial derivatives

$$
\left\{\begin{array}{l}
\frac{\partial \mathbf{U}}{\partial t}+\frac{\partial \mathcal{F}(\mathbf{U})}{\partial \breve{x}}=\mathbf{S}_{b} \\
\mathbf{U}(\breve{x}, t=0)= \begin{cases}\mathbf{U}_{\bar{\xi}}(\breve{x}) & \breve{x}<0 \\
\mathbf{U}_{\xi+1}(\breve{x}) & \breve{x}>0\end{cases}
\end{array}\right.
$$

where $\mathcal{F}=\mathbf{F} \cdot n_{x}+\mathbf{G} \cdot n_{y}$ is the projection of the flux onto the cell normal and $\mathbf{U}_{\xi}(\breve{x})$ and $\mathbf{U}_{\xi+1}(\breve{x})$ are the piecewise polynomial initial data for the DRP.

The solution of (1.5) is computed using the LFS solver in (Navas-Montilla \& Murillo, 2016) in combination with the Augmented Roe (ARoe) solver, which provides a suitable compromise between accuracy and computational cost. Furthermore, it allows to preserve the quiescent equilibrium.

\subsection{Energy cascade analysis}

When considering 2D turbulent flows, the analysis of the kinetic energy cascade can be a useful tool to determine whether the selected numerical scheme can provide trustworthy predictions or not. The numerical diffusion of the scheme can be assessed by using this tool. Theoretically, the 2D kinetic energy cascade in the inertial range for the system in (1.1) is of the form $\bar{E}(k) \propto k^{-3}$, where $E=\frac{1}{2}\left(u^{2}+v^{2}\right)$ is the kinetic energy function and $k$ the wave number (San \& Kara, 2015). An inverse energy cascade can also be observed at small wave numbers. 
To compute the numerical energy cascade, the kinetic energy is computed first in space, $E(x, y)$, at the desired time, and then transformed to the frequency domain using the Fast Fourier Transform (FFT), yielding $E\left(k_{x}, k_{y}\right)$. By an appropriate change to polar coordinates, we can express $E(k, \theta)$ where $k$ is the composite wave number, computed from $k_{x}, k_{y}$, and $\theta$ is the phase. To obtain a 1D representation of the energy cascade, a phase average of the kinetic energy is computed as follows

$$
\bar{E}(k)=\int_{0}^{2 \pi} E(k, \theta) d \theta
$$

\section{Numerical results}

All the problems considered here will be composed of a double shear layer (DSL), which involves the generation of the Kelvin-Helmholtz instability and will be hereafter referred to as DSL problems. Cyclic boundary conditions will be considered in all cases and a 1-st mode sinusoidal perturbation will be introduced (San \& Kara, 2015). The computation of this flow is of high complexity and requires a very accurate resolution of the fluxes in order to capture the generation and evolution of vortices at different scales.

The first test case concerns the resolution of the homogeneous DSL problem, the second case considers the DSL problem over varying bed and the third case the DSL problem over a frictional bed. The numerical results are assessed using the analysis of the energy cascade.

\subsection{DSL homogeneous problem}

The proposed scheme is here applied for the resolution of a 2D DSL problem without source term. Such flow is composed of two jets with initial velocity

$$
u(x, y, 0)= \begin{cases}-1 & 0.25<x<0.75 \\ 1 & \text { otherwise }\end{cases}
$$

and a constant initial depth of water $h(x, y, 0)=1$ over a flat and frictionless bed. The initial perturbation is given by $v(x, y, 0)=0.01 \sin (2 \pi x)$. Due to the inherent instability of the flow across the shear layer, the initial perturbation is amplified and evolves into a hydrodynamic two-dimensional turbulence.

The numerical solution is computed at $t=5 \mathrm{~s}$ using the 1-st, 3-rd and 5-th order ARL-ADER scheme, setting $\mathrm{CFL}=0.4$. Figure 1 shows the numerical solution for the instantaneous vorticity provided by such schemes in a $400^{2}$ cell grid. It is observed that only the 3-rd and 5-th order schemes are able to capture the turbulent shear layer within an acceptable level of accuracy, unlike the 1-st order scheme.

To analyse the mesh dependency of the solution, this test case has been computed in 3 different grids composed of $400^{2}, 500^{2}$ and $1000^{2}$ cells. The numerical solutions provided by the 3 -rd order scheme in such grids are depicted in Figure 2. It is observed that the use of a very fine grid $\left(1000^{2}\right)$ leads to the appearance of a complete 2D hydrodynamical turbulence where the vortical structures in the domain are fully interacting.

In this type of flow, a filamentation process is triggered by the small-scale vortices generated by the Kelvin-Helmholtz instability (San \& Kara, 2015). The degree of filamentation is higher as the order and the mesh size increases, as observed in Figure 1 and 2. The vortices interact to each other and are combined to form bigger vortices. This is observed in Figure 3, where the numerical vorticity computed by the 5 -th order scheme in a $400^{2}$ cell grid is shown at different times. 

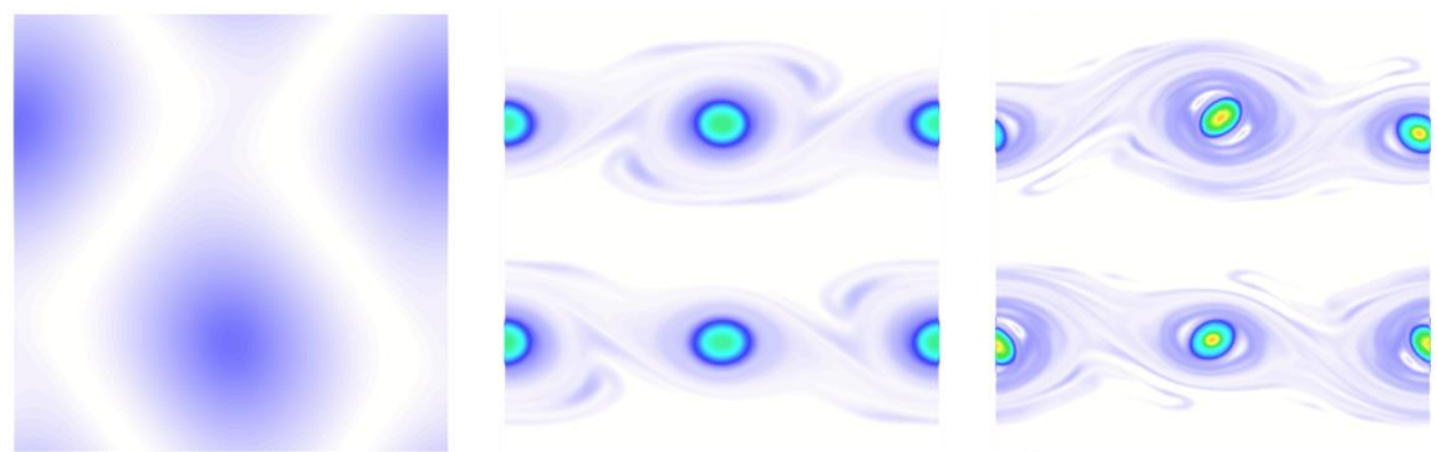

Figure 1: Instantaneous vorticity computed by the 1-st (left) and 3-rd (middle) and 5-th (right) order ARLADER schemes in a $400^{2}$ cell grid.
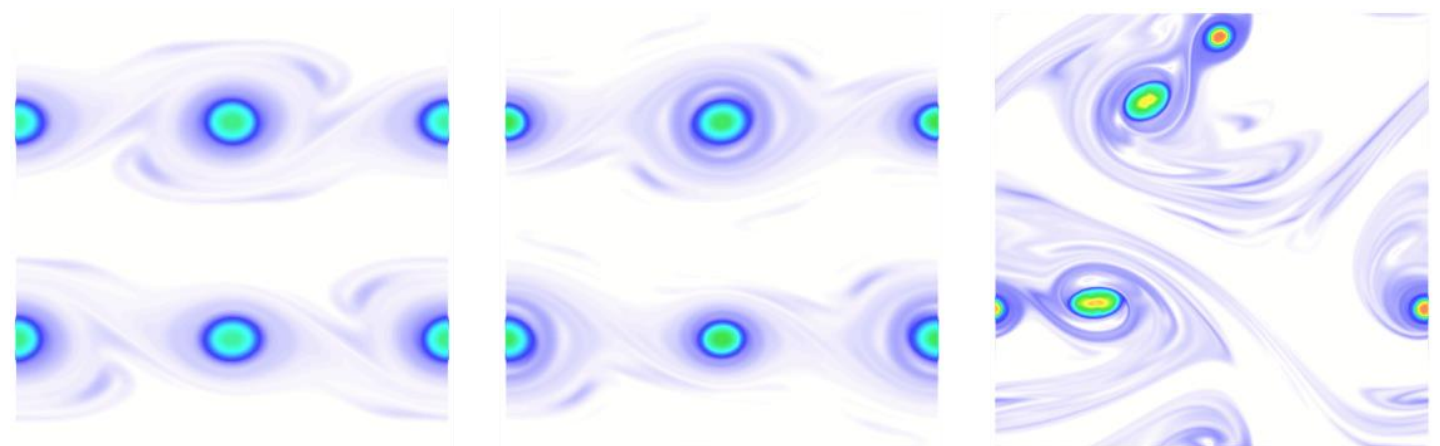

Figure 2: Instantaneous vorticity computed by the 3-rd order ARL-ADER scheme in a $400^{2}$ (left), $500^{2}$ (middle) and $1000^{2}$ cell grid (right).

A deeper analysis on the quality of the numerical solution for the resolution of $2 \mathrm{D}$ turbulence, in terms of the spurious non-physical (numerical) dissipation, can be carried out by plotting the energy cascade as outlined in Section 2.1. Figure 4 shows the energy cascade for such schemes computed in a $400^{2}, 500^{2}$ and $1000^{2}$ cell grid. It is evidenced again that the 1 -st order scheme is very diffusive and its inherent numerical dissipation cannot be reduced to an acceptable level with mesh refinement. On the other hand, the 3-rd and 5-th order schemes reproduce the inertial range of the theoretical energy cascade even when using the coarsest grid. Note that the differences between the numerical energy cascades provided by the 3-rd and 5-th order scheme are very small and are quickly reduced with mesh refinement, yielding the theoretical $\bar{E}(k) \propto k^{-3}$ dissipation rate. Such high order schemes are adequate to provide a trustworthy representation of the complex flow pattern in presence of $2 \mathrm{D}$ turbulence, even when using coarse grids. It is worth noting that an inverse energy cascade can also be identified at low wave numbers, though the transition (around $k=10$ ) is not clear due to the kind of source of excitation of the Kelvin-Helmholtz instability.

\subsection{DSL problem with bed elevation}

The proposed scheme is here applied to the resolution of the DSL problem over varying bed. The test case is configured as in Section 3.1, including now the effect of the bed elevation, given by 

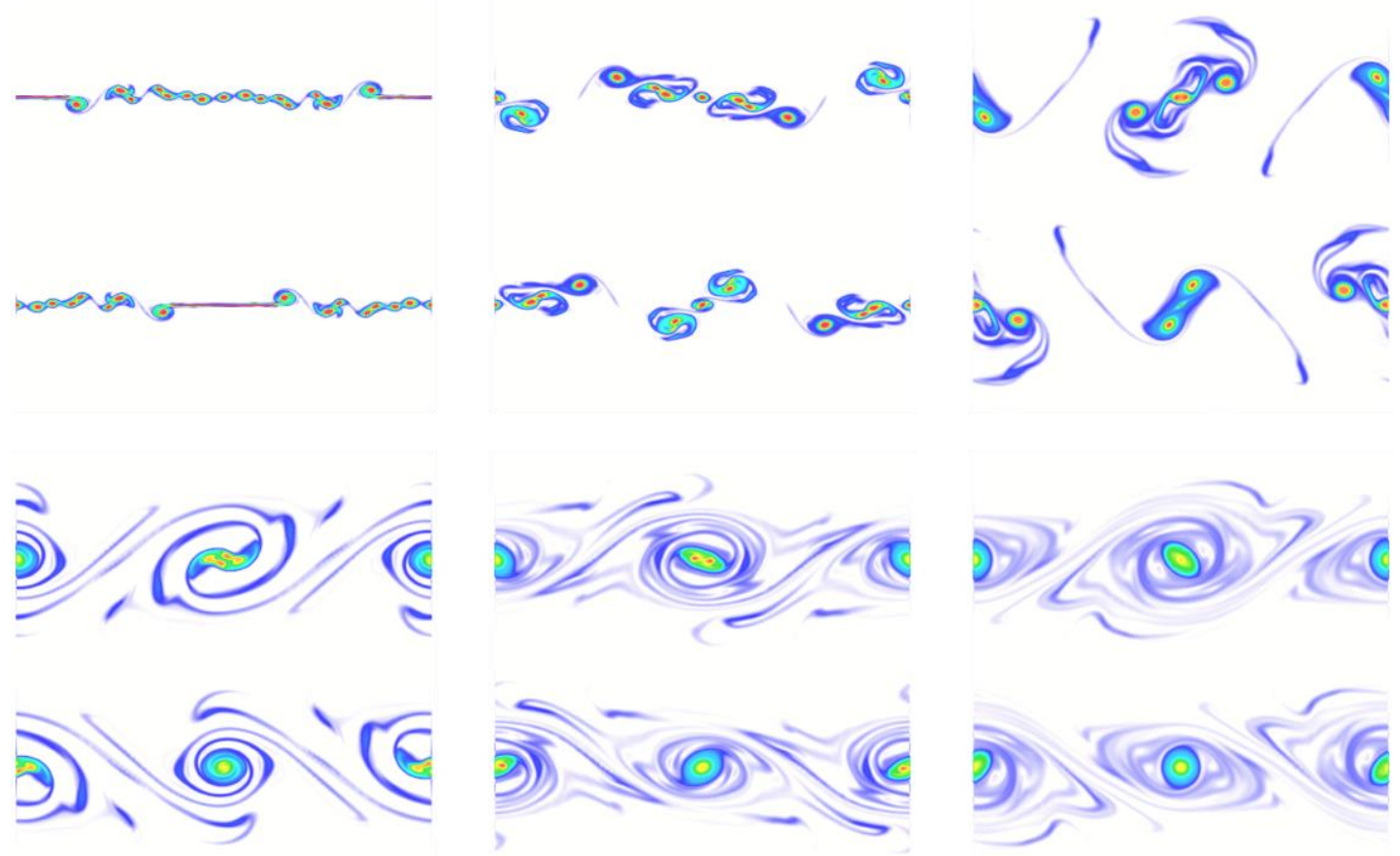

Figure 3: Instantaneous vorticity at $\mathrm{t}=0.3,0.5,1,1.5,2$ and 2.5 seconds computed by the 5 -th order ARLADER scheme in a $400^{2}$ grid.
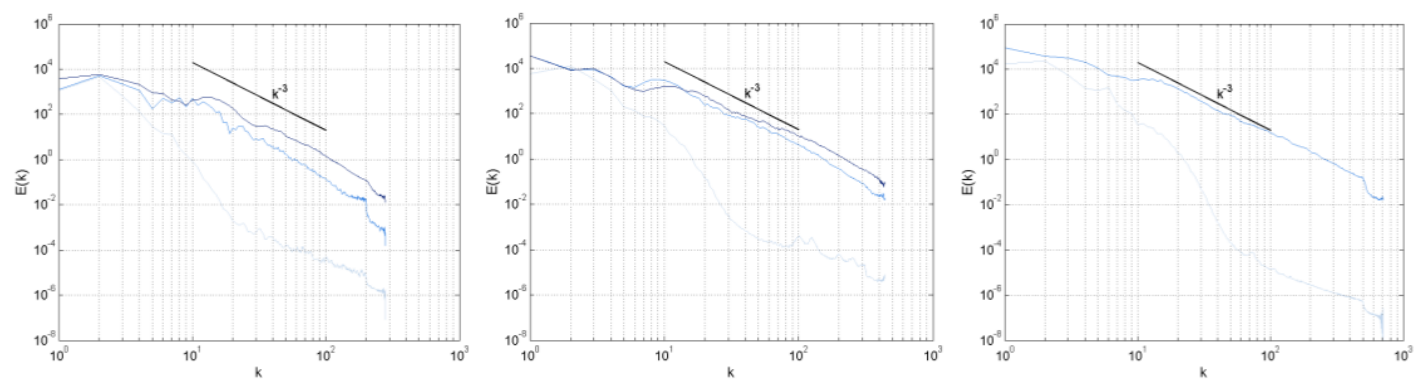

Figure 4: Kinetic energy cascade computed by the 1-st (-) and 3-rd (-) and 5-th (-) order ARL-ADER schemes in a $400^{2}$ (left), $500^{2}$ (middle) and $1000^{2}$ cell grid (right).

$z(x, y)=0.1 \sin (4 \pi(y-0.125))+0.1$. Such bed function only involves variations in the direction and allows to generate a flow pattern similar to that of Case 3.1, without large accelerations or shock formation, to reproduce the theoretical energy cascade. If using stronger bed variations, vertical accelerations become important, shocks may appear and the slope of the energy cascade decreases.

When considering soft variations, theoretically, for the system considered in (1.1), kinetic energy can be converted to pressure and potential energy and vice versa, without energy losses. Therefore, the kinetic energy cascade follows again the $k^{-3}$ rate. A 3D representation of the numerical water surface elevation, bed elevation and instantaneous vorticity at $t=3$ and $5 \mathrm{~s}$ is depicted in Figure 6 . 



Figure 5: Left: Kinetic energy cascade computed by the 1-st (-) and 3-rd (-) and 5-th (-) order ARL-ADER schemes in a $400^{2}$ cell grid. Right: Kinetic energy cascade computed by the 5-th (-) order ARL-ADER scheme for the case in $3.1(-)$ and $3.2(-)$.
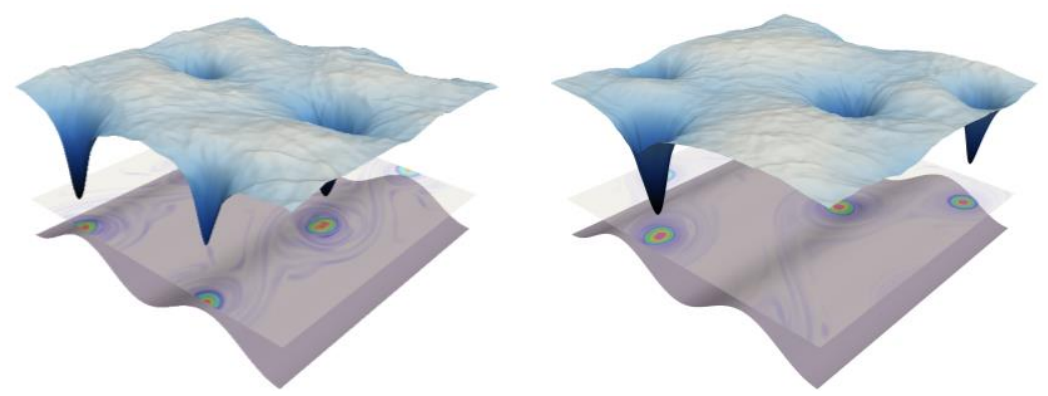

Figure 6: Water surface elevation, bed elevation and vorticity field computed by a 3-rd (left) and 5-th order scheme (right).

\subsection{DSL problem with friction}

The same configuration than in Section 3.1 is maintained, but setting now a non-zero friction source term. The friction coefficient is given by $c_{f}=g n^{2} h^{-1 / 3}$ where $n$ is the Manning coefficient.

Unlike in Sections 3.1 and 3.2 (soft bed variations), the energy cascade is now altered by the effect of bed friction, which involves energy dissipation. The numerical solution is computed in a $400^{2}$ cell grid using a 1-st, 3-rd and 5-th order ARL-ADER scheme. The numerical energy cascade is depicted in Figure 7 and shows the results for the homogeneous case, with $n=0$, as well as for the frictional case, with $n=0.1$ and $n=0.18$. When considering friction, it is observed that the dissipation rate is higher than the theoretical one. If compared to the homogeneous case, it seems that energy can be dissipated at any scale, but the highest differences are found for the smallest scales.

\section{Conclusions}

Complex turbulent flow patterns appear in presence of shear layers and are due to the KelvinHelmholtz instability. Such instability makes linear perturbations generate nonlinear turbulent structures that determine the mass exchange across the layers in the flow. In this work, we use a 2D extension of the arbitrary order ARL-ADER scheme to compute accurate predictions of mass exchange processes under a variety of scenarios involving turbulent shear layers over complex topographies with and without bed friction. The proposed scheme considers a suitable discretization of the source term to provide a physically based solution and to ensure the well-balanced property. 
The analysis of the kinetic energy cascade, computed using the numerical solution provided by the schemes, is used to assess the numerical performance of the numerical methods in terms of numerical diffusion.

It is known that the energy cascade for the $2 \mathrm{D}$ hydrodynamic turbulence possess an inertial range that decreases with $k^{-3}$ (in the incompressible and inviscid limit). The numerical results presented in this work show that the 3-rd and 5-th order schemes reproduce the theoretical results. On the other hand, the 1-st order scheme exhibits a much greater dissipation rate, even for fine meshes, which underscores the necessity of using high order schemes when seeking the numerical solution of turbulent flows within an affordable computational time.

When computing the kinetic energy cascade for the SWE with bed elevation, the theoretical slope of the inertial range is achieved if the bed variations are small. If stronger variations were considered, vertical accelerations and shock formation would involve transcendental alterations of the energy cascade. On the other hand, when computing the kinetic energy cascade for the SWE with bed friction, as expected, the overall energy level is lower than that of the non-frictional case, due to such an additional energy dissipation mechanism. Moreover, the slope of the inertial range of the cascade is greater than -3 .

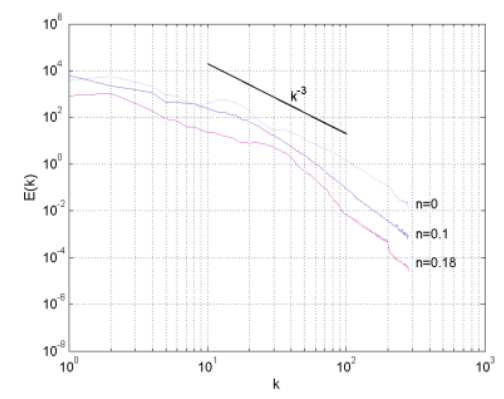

Figure 7: Kinetic energy cascade computed by the 5-th order ARL-ADER schemes in a $400^{2}$ cell grid for the frictionless case, with $n=0$, as well as $n=0.1$ and $n=0.18$.

\section{Acknowledgements}

The authors would like to acknowledge Dr. Omer San for the meaningful explanations on this topic and his previous work.

\section{References}

Castro, C., Toro, E., \& Käser, M. (2012). ADER scheme on unstructured meshes for shallow water: simulation of tsunami waves. Geophysical Journal International, 1505-1520.

Navas-Montilla, A., \& Murillo, J. (2016). Asymptotically and exactly energy balanced augmented flux-ADER schemes with application to hyperbolic conservation laws with geometric source terms. Journal of Computational Physics, 108-147.

San, O., \& Kara, K. (2015). Evaluation of Riemann flux solvers for WENO reconstruction schemes: Kelvin-Helmholtz instability. Computers and Fluids, 24-41.

Toro, E., \& Titarev, V. (2002). Solution of the generalised Riemann problem for advection-reaction equations. Proc. Roy. Soc. London, 196-215.

Vignoli, G., Titarev, V., \& Toro, E. (2008). ADER schemes for the shallow water equations in channel with irregular bottom elevation. Journal of Computational Physics, 2463-2480. 